\title{
MRI Directional Diffusivity Values of Cervical Cord White Matter: Multiple Sclerosis Patients Vs. Healthy Controls
}

\section{Sigal Tal'*, Hillel S. Maresky¹, Michael Abrahamy', Gili Givaty² and Nadav Berkovitz}

${ }^{1}$ Radiology Department, Assaf Harofeh Medical Center, Affiliated with Sackler School of Medicine Tel Aviv University, Israel ${ }^{2}$ Neurology Department, Sheba Medical Center, Affiliated with Sackler Faculty of Medicine Tel Aviv University, Israel

\section{Abstract}

Background: Early diagnosis of multiple sclerosis (MS) can lead to preventive treatment and a favorable prognosis. Cervical spinal cord matter that appears normal on conventional MR images may be detected as abnormal using advanced MRI techniques.

Objective: To pinpoint values and cervical spine locations in Diffusion Tensor Imaging (DTI) which best differentiate relapsing-remitting MS from healthy cases.

Methods: 10 relapsing-remitting MS and 8 healthy control patients underwent DTI of the cervical spine. DTI values were measured in anterior, lateral and posterior cord locations.

Results: Differences in values per location show statistical significance among all areas of the cord in both Normal Appearing White Matter (NAWM) and control groups. We derived an ADC cut-off value of $0.8 \mathrm{~mm}^{2} / \mathrm{sec}$ and $\lambda_{2}$ value of $0.8 \mathrm{~mm}^{2} / \mathrm{sec}$ for optimal differentiation between NAWM and control. Using these cut-off metrics, values above 0.8 for Receiver Operating Characteristics (ROC) (area under curve) may be considered 'good' tests for pathology. ROC analysis showed that the optimal differentiation between NAWM of MS patients versus healthy controls was seen in the anterior area of the cervical cord. Sensitivity and specificity for $\lambda_{2}$ and ADC are $85.7 \& 75.0$ and $85.7 \& 75.0$ respectively.

Conclusion: DTI can detect early changes in relapsing-remitting MS with the anterior area of the cervical spine being the most representative of disease processes. Diagnostic cut-off values should be considered.

Keywords: Magnetic resonance imaging; Diffusion tensor imaging; Multiple sclerosis; Relapsing-remitting; Spinal cord; Eigenvalue

Abbreviations: NAWM - Normal Appearing White Matter; MS: Multiple Sclerosis; WM: White Matter; RRMS: Relapsing-Remitting MS

\section{Introduction}

Multiple sclerosis (MS) is a chronic demyelinating disease of the central nervous system characterized by neurodegenerative and inflammatory processes that cause chronic disability. Axonal injury occurs in both acute inflammatory and chronic MS lesions [1,2].

The revised McDonald criteria include the spinal cord as a structure for diffusion in space. Asymptomatic spinal cord lesions are common in MS and uncommon in other white matter (WM) diseases [3]. The spinal cord lesion burden as obtained by a conventional MR examination (T1, T2, FLAIR) does not always correlate well with clinical disability or histological study [4]. Post-mortem pathological examination of the spinal cords of MS patients [5] show more extensive damage to the cord than what can be seen on MR imaging. Histopathological examination of the spinal cord reveals lesions that could not be detected on conventional MRI [3]. Therefore lesion quality or quantity is a problematic basis for MS diagnosis and treatment.

Diffusion Tensor Imaging (DTI) characterizes biological tissue microstructures by exploiting the quantification of water diffusion in tissues. DTI determines 3 perpendicular eigenvectors, given by their corresponding eigenvalues,

$\lambda_{1}, \lambda_{2}$, and $\lambda_{3}$. Longitudinal diffusivity $\lambda_{1}$ represents the water diffusivity parallel to the major axis of axonal fibers, which decreases in axonal injury. Diffusivity perpendicular to the axonal fibers, $\lambda_{2}$ and $\lambda_{3}$, is known as transverse diffusivity, and represents the extent of diffusion between adjacent myelin membranes. An increase in transverse diffusivity may reflect myelin injury and demyelination. Standard DTI metrics are derived from these eigenvalues. Fractional anisotropy (FA) and apparent diffusion coefficient (ADC) indices are derived from these 3 vectors [6]. ADC represents the average along the main axes. FA represents the degree of directionality of diffusion [7].

DTI metrics have been shown to differ for MS patients. The fractional anisotropy in the normal appearance lateral, posterior, and central cord was shown to be lower in MS patients than normal controls [8]. Spinal white matter regions that appear normal on conventional MR images, dubbed normal-appearing WM (NAWM), are also involved $[9,10]$. The corresponding DTI data of the cervical spine may be correlated with clinical disability and can predict the progression of the disease [11], and help evaluate therapeutic changes [12,13].

The sensory, motor, ascending and descending tracts of the spinal cord are anatomically different. Hence DTI values should vary between locations. In our study, we compared the cervical spinal cord white matter as a whole and the anterior, posterior, and lateral regions of interest (ROI) separately and compared DTI metrics

(FA, ADC, $\lambda_{1}, \lambda_{2} \lambda_{3}$ ), including the radial diffusivity (mean of $\lambda_{2} \& \lambda_{3}$ ).

Our goals were to pinpoint the ROIs that best differentiated between NAWM in MS patients and normal WM in healthy controls and to provide a statistically robust DTI value useful in facilitating this differentiation.

*Corresponding author: Sigal Tal, Radiology Department, Assaf Harofeh MedicalCenter Affiliated to Sackler Faculty of Medicine Tel Aviv, University Tel Aviv, Israel, Zerifin 70300 Israel, Tel: +972-50-519-1258; E-mail: sigalt@asaf.health.gov.il

IReceived July 05, 2015; Accepted August 17, 2015; Published August 19, 2015

Citation: Tal S, Maresky HS, Abrahamy M, Givaty G, Berkovitz N (2015) MR Directional Diffusivity Values of Cervical Cord White Matter: Multiple Sclerosis Patients Vs. Healthy Controls. J Spine 4: 246.doi:10.4172/21657939.1000246

Copyright: (c) 2015 Tal S, et al. This is an open-access article distributed under the terms of the Creative Commons Attribution License, which permits unrestricted use, distribution, and reproduction in any medium, provided the original author and source are credited. 


\section{Methods}

\section{Subjects}

Ten patients with relapsing-remitting MS (RRMS) were referred to our hospital and underwent an MRI scan with DTI included in the study protocols. At the time of imaging, there was no clinical suspicion of an acute MS attack. The mean age was 44 years (range, 18-65; SD 15); 7 females and 3 males. We compared their data to a control group composed of 8 healthy patients that underwent non-pathological MRI scans with DTI included in the protocols. The mean age was 44 years (range, 22-50; SD 12); 4 females and 4 males. All MRI examinations were conducted using the same MRI system and the same DTI protocol parameters. IRB approval was granted for the study. Demographic data for the RRMS group appears in Table 1.

\section{MR imaging}

Imaging of the cervical spine was performed at $1.5 \mathrm{~T}$ (Siemens Magnetom Aera). Sagittal and coronal T1-weighted imaging was obtained followed by turbo spin-echo sagittal T1- and T2-weighted imaging, axial turbo spin-echo T2-weighted and spin-echo T1-weighted imaging. Axial DTI of the entire cervical spinal cord was performed, using pulsed gradient, spin-echo, echo-planar imaging. Repetition time (TR) 6500, echo time (TE) 83; Matrix 128 X 128; Field of view $230 \mathrm{~mm}$ X $230 \mathrm{~mm}$; Contiguous slice thickness $3 \mathrm{~mm} ; 2$ b-values $0 \& 1000 \mathrm{~s} /$ $\mathrm{mm}^{2}$; acquisition time $7.04 \mathrm{~min}$. Diffusion weighting was applied along 30 non collinear axes, pixel size $1.8 \times 1.8 \mathrm{~mm}^{2}$.

\section{Image processing}

Quantitative analysis of the DTI data and DTI maps were generated by the DTI task card using MRWP with a SyngoMR D11 imaging software platform (Siemens Medical Solutions, Erlangen, Germany).

\section{Data and statistical analysis}

A ROI sampling method previously described by Hasseltine et al. [8] was used. For both groups, measurements were taken from axial DTI images at the C2-C3 level. No cases had MS lesions at this level.

\begin{tabular}{|l|c|c|}
\hline & Control $(\mathbf{n}=\mathbf{8})$ & MS $(\mathbf{n}=\mathbf{1 0})$ \\
\hline Age (years) & $41 \pm 12(22-50)$ & $44 \pm 15(18-65)$ \\
\hline Gender & $\mathrm{F}=7$ & $\mathrm{~F}=4$ \\
\hline & $\mathrm{M}=3$ & $\mathrm{M}=4$ \\
\hline Disease Duration (years) & $\mathrm{N} / \mathrm{A}$ & 4.2 \\
\hline Median EDSS & $\mathrm{N} / \mathrm{A}$ & 2 \\
\hline
\end{tabular}

Table 1: Subject demographics and clinical characteristics.

\begin{tabular}{|c|c|c|c|c|c|c|}
\hline DTI & \multicolumn{3}{|c|}{ Control } & \multicolumn{3}{c|}{ NAWM } \\
\hline \multirow{2}{*}{ Parameters } & Ant & Pos & Lat & Ant & Pos & Lat \\
\cline { 2 - 7 } & $\mathrm{n}=14$ & $\mathrm{n}=14$ & $\mathrm{n}=14$ & $\mathrm{n}=20$ & $\mathrm{n}=20$ & $\mathrm{n}=20$ \\
\hline$\lambda_{1} \times 10^{-3}\left(\mathrm{~mm}^{2} /\right.$ & 1.71 & 2.00 & 1.67 & 1.80 & 2.34 & 1.99 \\
$\mathrm{sec})$ & $(0.16)$ & $(0.16)$ & $(0.24)$ & $(0.31)$ & $(0.35)$ & $(0.37)$ \\
\hline$\lambda_{2} \times 10^{-3}\left(\mathrm{~mm}^{2} /\right.$ & 0.40 & 0.57 & 0.71 & 0.51 & 0.78 & 0.96 \\
$\mathrm{sec})$ & $(0.09)$ & $(0.09)$ & $(0.09)$ & $(0.20)$ & $(0.27)$ & $(0.21)$ \\
\hline$\lambda_{3} \times 10^{-3}\left(\mathrm{~mm}^{2} /\right.$ & 0.30 & 0.44 & 0.64 & 0.26 & 0.41 & 0.56 \\
$\mathrm{sec})$ & $(0.12)$ & $(0.20)$ & $(0.20)$ & $(0.08)$ & $(0.08)$ & $(0.07)$ \\
\hline $\mathrm{ADC}^{2} 10^{-}$ & 0.84 & 1.19 & 1.20 & 0.79 & 0.99 & 0.98 \\
${ }^{3}\left(\mathrm{~mm}^{2} / \mathrm{sec}\right)$ & $(0.24)$ & $(0.23)$ & $(0.19)$ & $(0.09)$ & $(0.08)$ & $(0.11)$ \\
\hline $\mathrm{FA}$ & 0.74 & 0.71 & 0.52 & 0.78 & 0.73 & 0.55 \\
& $(0.08)$ & $(0.07)$ & $(0.12)$ & $(0.05)$ & $(0.07)$ & $(0.07)$ \\
\hline $\mathrm{RD} \times 10-$ & 0.41 & 0.61 & 0.80 & 0.33 & 0.49 & 0.64 \\
${ }^{3}\left(\mathrm{~mm}^{2} / \mathrm{sec}\right)$ & $(0.14)$ & $(0.21)$ & $(0.16)$ & $(0.08)$ & $(0.08)$ & $(0.07)$ \\
\hline
\end{tabular}

Table 2: Diffusion tensor imaging (DTI) values of white matter regions of interest (ROIs) in different areas.

Ant: Anterior; Pos: Posterior; Lat: Lateral. Standard deviation in parentheses.

\begin{tabular}{|l|c|c|c|c|c|c|}
\hline \multirow{2}{*}{ DTI Parameters } & Control & Control & Control & NAWM & NAWM & NAWM \\
\cline { 2 - 7 } & $\begin{array}{c}\text { Ant vs. } \\
\text { Lat }\end{array}$ & $\begin{array}{c}\text { Pos vs. } \\
\text { Lat }\end{array}$ & $\begin{array}{c}\text { Ant vs. } \\
\text { Pos }\end{array}$ & $\begin{array}{c}\text { Ant vs. } \\
\text { Lat }\end{array}$ & $\begin{array}{c}\text { Pos vs. } \\
\text { Lat }\end{array}$ & $\begin{array}{c}\text { Ant vs. } \\
\text { Pos }\end{array}$ \\
\hline$\lambda_{1}$ & 0.0003 & $<0.0001$ & 0.6328 & 0.0764 & $<0.0001$ & 0.0048 \\
\hline$\lambda_{2}$ & 0.0003 & $<0.0001$ & $<0.0001$ & $<0.0001$ & 0.0009 & 0.0271 \\
\hline$\lambda_{3}$ & $<0.0001$ & $<0.0001$ & $<0.0001$ & $<0.0001$ & 0.0114 & 0.0031 \\
\hline ADC & 0.7904 & $<0.0001$ & $<0.0001$ & $<0.0001$ & $<0.0001$ & 0.8708 \\
\hline FA & $<0.0001$ & 0.0353 & $<0.0001$ & $<0.0001$ & 0.2011 & $<0.0001$ \\
\hline RD & $<0.0001$ & $<0.0001$ & $<0.0001$ & $<0.0001$ & 0.0008 & 0.0027 \\
\hline Ratio & $<0.0001$ & 0.0137 & $<0.0001$ & 0.0002 & 0.1378 & $<0.0001$ \\
\hline
\end{tabular}

Table 3: Differences in DTI metric values based on different areas within the Control and NAWM Populations.

Ant: Anterior; Pos:Posterior; Lat: Lateral.

\begin{tabular}{|c|c|c|c|}
\hline Area Under Curve & Ant & Pos & Lat \\
\hline$\lambda 1$ & 0.76 & 0.78 & 0.55 \\
\hline$\lambda 2$ & 0.85 & 0.78 & 0.75 \\
\hline$\lambda 3$ & 0.71 & 0.48 & 0.6 \\
\hline ADC & 0.81 & 0.79 & 0.64 \\
\hline FA & 0.6 & 0.59 & 0.66 \\
\hline
\end{tabular}

Table 4: Area under curve for ROC analysis of DTI metrics per area. Ant: Anterior; Pos: Posterior; Lat: Lateral

As in Hassteltine et al., a central spinal cord circular area was marked to standardize the placement of measurement Region of Interest (ROIs) and indicate an area of mixed grey and white matter measurements. Similarly to approximate the known locations of the spinothalamic tracts of both the anterior and posterior columns, and to ensure the consistent placement of ROIs, the anterior and posterior ROIs were placed in a para-midline location. Distance was maintained between the ROIs and the edge of the cord to minimize volume averaging with the adjacent CSF. The lateral ROIs were placed to approximate the location of the corticospinal tracts. Thus, measurements were taken from 6 ROIs: two anterior, two laterals, and two posterior, linked with separate anatomical areas of the cervical spinal cord white matter. All measurement-ROIs were of uniform size. Measurements were taken of FA, ADC, $\lambda_{1}, \lambda_{2}$ and $\lambda_{3}$. Additionally radial diffusivity (RD: mean of $\lambda_{2}$ and $\lambda_{3}$ ) was calculated from these values.

A receiver operating characteristic (ROC) curve and the area under the curve was calculated for each parameter and each area of cervical cord to find the optimal test to differentiate between MS pathology and control.

The data were analyzed using the SAS ${ }^{\circledast}$ version 9.1 (SAS Institute, Cary North Carolina). All variables and parameters were tabulated by descriptive statistics. The tables report the appropriate tests; sample size, absolute and relative frequency and 95\% confidence interval (CI). All T-tests were two-tailed, and a p-value of $5 \%$ or less was considered statistically significant.

\section{Results}

Differences in spinal DTI metrics (Table 2) between anterior, posterior and lateral areas of the cord show statistically significance among all areas of the cord (Table 3). We derived an ADC cut-off value of $1.08 \mathrm{~mm}^{2} / \mathrm{sec}$ and $\lambda_{2}$ value of $0.8 \mathrm{~mm}_{2} / \mathrm{sec}$ for optimal differentiation between NAWM and control. Using these cutoff metrics (ADC and $\lambda_{2}$ ), values above 0.8 for ROC (area under curve) may be considered 'good' tests for pathology (Table 4). Higher values than the cut-off are suspicious of NAWM pathology. Comparing each area between the populations, statistically significant differences are noted in select areas (Table 5). Derived sensitivity and specificity for $\lambda_{2}$ and ADC in the anterior area are $85.7 \& 75.0$ and $85.7 \& 75.0$ respectively. 


\begin{tabular}{|c|c|c|c|}
\hline \multirow{2}{*}{ DTI Parameters } & \multicolumn{3}{|c|}{ Control vs. NAWM } \\
\cline { 2 - 4 } & Ant & Pos & Lat \\
\hline$\lambda_{1}$ & 0.0078 & 0.0008 & 0.2839 \\
\hline$\lambda_{2}$ & $<0.0001$ & 0.0029 & 0.0338 \\
\hline$\lambda_{3}$ & 0.1177 & 0.5583 & 0.2815 \\
\hline ADC & 0.0007 & 0.0019 & 0.4103 \\
\hline FA & 0.4287 & 0.4388 & 0.1318 \\
\hline RD & 0.0005 & 0.0241 & 0.0578 \\
\hline Ratio & 0.6693 & 0.8829 & 0.6002 \\
\hline
\end{tabular}

Table 5: Comparison of DTI metric values between NAWM and controls based on different areas. 2 tailed T-tests.

Ant: Anterior; Pos: Posterior; Lat: Lateral

In both the NAWM and control groups, longitudinal diffusivity, $\lambda_{1}$, tended to be highest in the posterior spinal areas. Lateral areas had the lowest transverse diffusivity values. FA was the lowest in the anterior areas and ADC in the lateral areas. Radial diffusivity was highest in the posterior areas.

When the spinal tract was treated as a whole, significant differences were noted in $\lambda_{1}, \lambda_{2}, A D C$ and RD between NAWM and controls in the anterior and posterior areas. For the lateral areas only $\lambda_{2}$ showed a significant change. There was no correlation between DTI metrics and EDSS or disease duration.

\section{Discussion}

Conventional MR is routinely used to detect spinal cord lesions in patients with MS. However, conventional MR scan findings are poorly correlated with the clinical presentation of MS patients [10]. Previous studies have failed to find a correlation between the clinical disability of MS patients and the number and extent of spinal cord lesions detected by MR [4]. We demonstrate the use of DTI as a complement to MS detection and description (Figure 1).

It was argued that the pathological features of the NAWM of MS patients may relate to a low-grade inflammatory and demyelinating process with resultant edema, demyelination, cellular infiltration, gliosis and axonal loss. Myelin and axonal loss are considered to contribute the most to these DTI changes [2]. A quantitative postmortem study [14] found a significant reduction in axonal density that grossly appears normal in MS patients. Previously undetected lesions in NAWM have been reported in 7 Tesla MRI studies [15].

Cercignani et al. [16] indicated that for RRMS patients and secondary progressive MS, the mean diffusivity measurements in brain tissue could mark the evolution of the disease. They found an increase in isotropic diffusion and a decrease in anisotropic diffusion in NAWM. In a recent study [17] FA was shown to be reduced in MS spine lesions (and in neuromyelitis optica) compared to controls, with increased radial diffusivity. A clinical study of DTI data and average EDSS values [17] found a significant inverse correlation between FA values of the posterior column in C2 and EDSS (Pearson $r=-0.800, p$ $=0.017)$. This would suggest a possible role for DTI values as a marker of clinical disability.

Our study evaluated DTI changes and DTI-derived metrics of the cervical spinal cord of NAWM in RRMS patients per area of cord (anterior, posterior and lateral). This data then served to compare differences among these anatomical areas measured with separate ROIs, as recent literature has shown some differences between tracts $[8,17]$.

Differences in the eigenvalues between NAWM and controls for both longitudinal and transverse diffusivity were found. This pattern resembles Wallerian degeneration which is characterized by axonal injury, followed by myelin disruption [18]. In studies of experimental
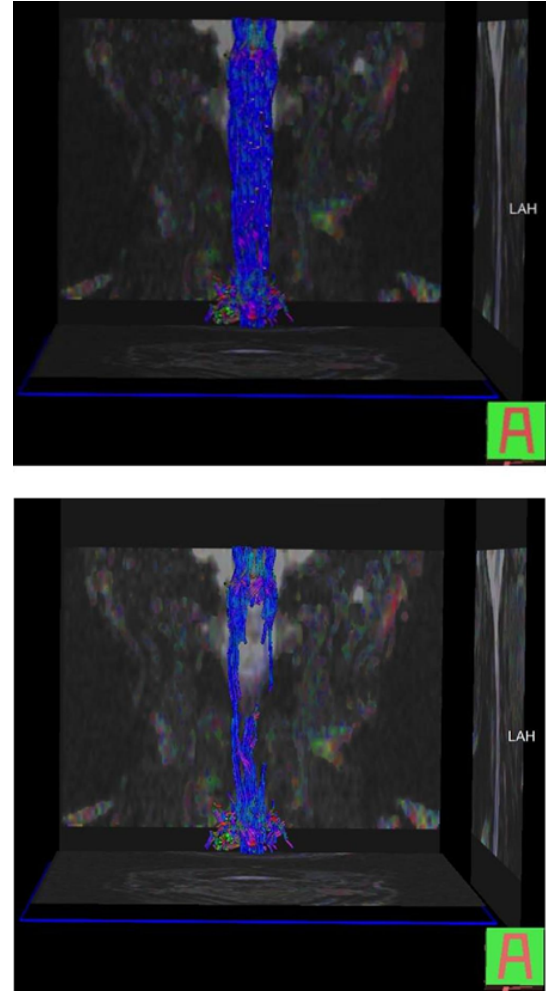

Figure 1: Reconstruction of Spine DTI tractography: a) Normal vs. (b) patient with MS. Note the splaying of blue fibres around the MS lesion.

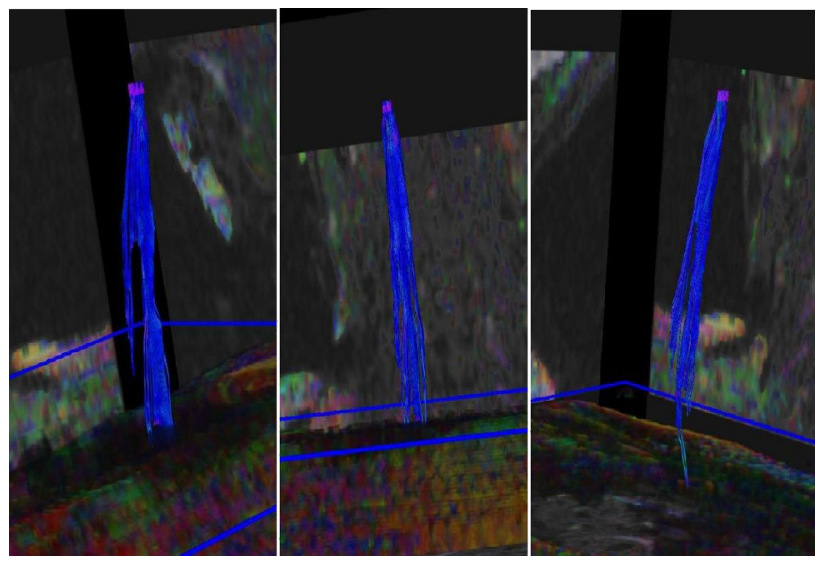

Figure 2: Oblique views of DTI images.

autoimmune encephalomyelitis-affected mouse spinal cords, longitudinal diffusivity and transverse diffusivity correlated with axonal injury and demyelination, respectively [19].

One of the goals of MRI, and more specifically diffusion imaging in MS, is to identify and monitor disease progression and assess the efficacy of different treatments. Baseline cross-sectional areas of the cervical cord and FA in the cord correlated with increased disability at 2.4 years follow-up [11]. Freund et al. emphasized the role of transverse diffusivity and its dynamic changes over time [13]. Lower radial diffusivity of the cortico-spinal tract at baseline was associated with better clinical outcome. As patients improved clinically during the follow-up, they showed a growing decrease in radial diffusivity of the cortico-spinal tract (Figure 2). This aligns with our results 
between NAWM and control cases. The authors suggested that this change reflects processes in the spinal cord including the resolution of inflammation and re-myelination contributing to clinical recovery in MS.

The difference in locations between the different cord areas and different ROIs is important to DTI metrics and their baseline values. They should be measured and compared as separate entities.

The ability of two continuous variables to diagnose an outcome can be compared using ROC curves and the area under the curve [20]. The characteristics of a ROC plot provide an index of accuracy by demonstrating the ability to discriminate between alternative states of health [21]. As the area under the curve of the ROC plot is a measure of the overall performance of a diagnostic test, the greater the area under the curve, the better the overall performance of the diagnostic test [22]. Though various methods have been described to capture discrimination, the area under the ROC curve is the most popular [23].

ROC analysis showed that the optimal differentiation between pathological NAWM in the MS group versus the control group was detected in the anterior areas. Anterior ADC and $\lambda_{2}$ showed the highest sensitivity and specificity. Conversely there were metrics with a calculated ROC below 0.5 that could not be used effectively as a disease defining criteria. Though FA has been shown to be lower in MS patients [17] the area under the curve was low for all areas and thus not an effective tool for MS diagnosis. A suggested cut-off defining value for ADC was $0.8 \mathrm{~mm}^{2} / \mathrm{sec}$ and for $\lambda_{2}$ was $0.8 \mathrm{~mm}^{2} / \mathrm{sec}$. One limitation of our study is the small study group sizes which were not large enough to properly examine correlations between parameters and clinical disability. A larger study population would enable tailoring of DTI values by age, sex or other significant criteria with a subsequent improvement in sensitivity and specificity of cut-off values. Another limitation exists in that there is no histopathological correlation of the MRI findings and is based on MRI DTI protocols in our small pilot study. Further large groups study should be performed to examine for the predictive value of the metrics, and to evaluate the impact of age related vascular differences in patients with MS [24]. Conversely, the statistical significance of the findings in a small population study further emphasizes the variance between MS and control DTI metrics.

The spinal cord tracts are anatomically different between the different areas with different fibre thickness and function [4]. Similarly MS lesions are known to have an unequal spread withing the cord with a preference for the central cord, particularly the posterior elements [4]. The development of a 'good' test for pathology based on findings with ROC values above 0.8 can provide a statistically robust differentiation in specific cervical cord areas between MS patients and healthy controls. As such, DTI metrics in the NAWM could become a defining criterion of disease.

\section{Conclusion}

Multiple sclerosis DTI values of the cervical spinal cord should include evaluation based specific areas within the cord (anterior, posterior and lateral). We suggest a cut-off value of disease in NAWM was $0.8 \mathrm{~mm}^{2} / \mathrm{sec}$ for ADC and $0.8 \mathrm{~mm}^{2} / \mathrm{sec}$ for $\lambda_{2}$. ROC analysis showed these values as 'significant' tests for pathology detection.

\section{Conflict of Interest}

The authors have no conflict of interest to disclose.

IRB approval was given for the study.

\section{References}

1. Trapp BD, Peterson J, Ransohoff RM, Rudick R, Mork S, et al. (1998) Axonal transection in the lesions of multiple sclerosis. N Engl J Med 338: 278-85.
2. Hesseltine SM, Ge Y, Law M (2007) Applications of diffusion tensor imaging and fiber tractography. Appl Radiol 36: 8-23.

3. Bot JC, Blezer EL, Kamphorst W, Lycklama A Nijeholt GJ, et al. (2004)The spinal cord in multiple sclerosis: relationship of high-spatial-resolution quantitative MR imaging findings to histopathologic results. Radiology 233: 531-40.

4. Cruz LC, Domingues RC, Gasparetto EL (2009) Diffusion tensor imaging of the cervical spinal cord of patients with relapsing-remising multiple sclerosis: a study of 41 cases. Arq Neuropsiquiatr 67: 391-395.

5. Gilmore CP, Donaldson I, Bo L, Owens T, Lowe J, Evangelou N (2009) Regional variations in the extent and pattern of grey matter demyelination in multiple sclerosis: a comparison between the cerebral cortex, cerebellar cortex, deep grey matter nuclei and the spinal cord. J Neurol Neurosurg Psychiatry 80: 182-187.

6. Sigal T, Shmuel M, Mark D, Gil H, Anat A. (2012) Diffusion tensor imaging of corpus callosum integrity in multiple sclerosis: correlation with disease variables. J Neuroimaging 22: 33-37.

7. Ge Y (2006) Multiple sclerosis: the role of MR imaging. AJNR Am J Neuroradiol. 27: $1165-1176$

8. Hesseltine SM, Law M, Babb J, Rad M, Lopez S, et al. (2006) Diffusion tenso imaging in multiple sclerosis: assessment of regional differences in the axia plane within normal-appearing cervical spinal cord. AJNR Am J Neuroradiol 27: 1189-1193.

9. Rovaris M, Judica E, Gallo A, Benedetti B, Sormani MP, et al. (2006) Grey matter damage predicts the evolution of primary progressive multiple sclerosis at 5 years. Brain 129: 2628-2634.

10. Miller DH, Grossman RI, Reingold SC, McFarland HF (1998) The role of magnetic resonance techniques in understanding and managing multiple sclerosis. Brain 121: 3-24.

11. Agosta F, Absinta M, Sormani MP, Ghezzi A, Bertolotto A, et al. (2007) In vivo assessment of cervical cord damage in MS patients: a longitudinal diffusion tensor MRI study. Brain 130: 2211-2219.

12. Jones JG, Cen SY, Lebel RM, Hsieh PC, Law M (2013) Diffusion tensor imaging correlates with the clinical assessment of disease severity in cervical spondylotic myelopathy and predicts outcome following surgery. AJNR Am J Neuroradiol 34: 471-478

13. Freund $P$, Wheeler-Kingshott $C$, Jackson J, Miller D, Thompson A, et al. (2010) Recovery after spinal cord relapse in multiple sclerosis is predicted by radial diffusivity. Mult Scler 16: 1193-1202.

14. Evangelou N, Esiri MM, Smith S, Palace J, Matthews PM (2000) Quantitative pathological evidence for axonal loss in normal appearing white matter in multiple sclerosis. Ann Neurol 47: 391-395.

15. Mistry N, Tallantyre EC, Dixon JE, Galazis N, Jaspan T, et al. (2011) Focal multiple sclerosis lesions abound in 'normal appearing white matter'. Mult Scler 17: 1313-1323.

16. Cercignani M, Inglese M, Pagani E, Comi G, Filippi M (2001) Mean diffusivity and fractional anisotropy histograms of patients with multiple sclerosis. AJNR Am J Neuroradiol 22: 952-958.

17. Pessoa FM, Lopes FC, Costa JV, Leon SV, Domingues RC, et al. (2012) The cervical spinal cord in neuromyelitis optica patients: a comparative study with multiple sclerosis using diffusion tensor imaging. Eur J Radiol 81: 2697-2701.

18. Simon JH, Kinkel RP, Jacobs L, Bub L, Simonian N (2000) A Wallerian degeneration pattern in patients at risk for MS. Neurology 54: 1155-1160.

19. Klawiter EC, Xu J, Naismith RT, Benzinger TL, Shimony JS, et al. (2012) Increased radial diffusivity in spinal cord lesions in neuromyelitis optica compared with multiple sclerosis. Mult Scler 18: 1259-1268.

20. Bewick V, Cheek L, Ball J (2004) Statistics review 13: receiver operating characteristic curves. Crit Care 8: 508-512.

21. Zweig MH and Campbell G (1993) Receiver-operating characteristic (ROC) plots: a fundamental evaluation tool in clinical medicine. Clin Chem 39: 561-577.

22. Park SH, Goo JM, Jo CH (2004) Receiver operating characteristic (ROC) curve: practical review for radiologists. Korean J Radiol 5: 11-18.

23. Cook NR (2008) Comments on 'Evaluating the added predictive ability of a new marker: From area under the ROC curve to reclassification and beyond' by M. J. Pencina et al., Statistics in Medicine. Stat Med 27: 191-195.

24. Ciciarello F, Mandolesi S, Galeandro AI, Marceca A, Rossi M, et al. (2004) Agerelated vascular differences among patients suffering from multiple sclerosis. Current neurovascular research 11: 23-30. 